\title{
PKA Regulatory Subunit RIalpha Mixed- Backbone Antisense Oligonucleotide GEM 231
}

National Cancer Institute

\section{Source}

National Cancer Institute. PKA Regulatory Subunit Rlalpha Mixed-Backbone Antisense

Oligonucleotide GEM 231. NCI Thesaurus. Code C1810.

A mixed backbone oligonucleotide exhibiting antitumor activity. GEM-231 is complementary to the RI alpha subunit of Protein Kinase A (PKA), resulting in downregulation of PKA expression and ultimately tumor growth. $(\mathrm{NCl})$ 